\title{
Matchmaking for Autonomous Agents in Electronic Marketplaces
}

Daniel Veit ${ }^{1}$

Jörg P. Müller ${ }^{2}$

Martin Schneider ${ }^{3}$
Björn Fiehn ${ }^{4}$

\begin{abstract}
Matchmaking is the process of mediating demand and supply based on profile information. Matchmaking plays a crucial role in agent-based electronic marketplaces: the problem to be solved is to find the most appropriate agents, products, or services for a task, negotiation, or market transaction. Most real-world problems require multidimensional matchmaking, i.e., the ability to combine various dimensions of decision-making to define an overall solution to a matchmaking problem, requiring the interplay of multiple matchmaking algorithms. In addition, in order to be applicable for real-world applications, the matchmaking component must be easily integrated into standard industrial marketplace platforms.

The work described in this work aims at deploying agent-based matchmaking for industrial electronic business applications. The main contributions of this work are the following: (i) we provide a configurable framework called GRAPPA (Generic Request Architecture for Passive Provider Agents) which is designed to be adapted to electronic marketplace applications. Using GRAPPA, system designers can easily specify demand and supply profiles as XML objects; (ii) within GRAPPA we provide an extensible library of matchmaking functions (building blocks) that can be used for rapid development of matchmaking solutions that include standard information retrieval algorithms.
\end{abstract}

Areas: artificial market systems and electronic commerce, middle agents, agent-based software engineering

Keywords: Matchmaking, Distance Computation, Distance Functions, Marketplaces, XML, Enterprise Java Beans ${ }^{\mathrm{TM}}$

\section{INTRODUCTION}

Electronic business is taking off, and autonomous agents are likely to play an important role in the next generation of electronic business systems. In these systems, electronic marketplaces will be constructed where interactions and transactions that are today carried out by humans (or by machines, based on simple EDI) will be performed increasingly by software agents, acting on behalf of humans or organizations. However, advancing today's agentbased marketplaces (e.g., [5]) to industrial applicability, is a long way and will require a rich and secure infrastructure.

Copyright $2001 \mathrm{ACM}$. This is the author's version of the work. It is posted here for your personal use. Not for redistribution. The definitive Version of Record can be found at

https://doi.org/10.1145/375735.375874
Firstly, agents and agent functions need to be integrated into stateof-the art industrial marketplace platforms, Enterprise Information and ERP systems. Secondly, for an agent-based marketplace to thrive, additional facilitator services are required to provide mechanisms that allow agents to find the marketplace, service, product, or information they want (see [3]).

- Demand and supply profiles in electronic marketplaces are often complex (e.g., matching job profiles against applicant profiles) and require multi-dimensional matchmaking. Often, a combination of existing methods is adequate to deal with different aspects of matchmaking.

- Demand and supply information is distributed and heterogeneous. Thus, distributed search and ontology mapping may be required to achieve comparable profiles.

- Demand and supply profiles differ depending on the application domain; also the underlying business logic to determine the quality of a match (relevance functions) is very domain-specific in part. The framework must support this variety.

We found that existing matchmaking approaches (see [1],[2],[4],[6],[7],[9] and [10]) do not appropriately address these requirements. Therefore, we designed and built a configurable matchmaking framework called GRAPPA (Generic Request Architecture for Passive Provider Agents) for electronic marketplace applications.

\section{AGENT BASED MATCHMAKING}

Matchmaking is not only a key task in multi-agent systems, it is also a crucial function in industrial portals and on electronic marketplaces. The provider who will enable the most effective matches between demand and supply will gain a competitive advantage and increase the acceptance and popularity of their marketplaces.

We understand matchmaking as a function which accepts as input a set of offers (candidate profiles) and a request (centroid profile)

1 University Karlsruhe (TH), Information Management and Systems, Englerstr. 14, 76131 Karlsruhe, Germany,

+49-721-608-8372, Daniel.Veit@iw.uni-karlsruhe.de

2 Siemens AG CT, Intelligent Autonomous Systems, OttoHahn-Ring 6, 81730 Munich, Germany,

+49-89-636-43135, Joerg.Mueller@mchp.siemens.de

3 Siemens AG CT , Intelligent Autonomous Systems, OttoHahn-Ring 6, 81730 Munich, Germany, +49-89-636-44257, Martin.Schneider@mchp.siemens.de

4 Philipps-University Marburg, Department of Mathematics and Computer Science, Hans-Meerwein Str., 35032

Marburg, Germany, +49-6421-28-21513,

Fiehn@informatik.uni-marburg.de 
and provides as output a ranked list of the $k$ best offers with respect to the request. Candidate and centroid profiles are multidimensional objects possibly providing the service description of agents. In GRAPPA the process of matchmaking is referred to as a parallel computation of sub-relevance values for each dimension of the candidate and the centroid profile where a mapping maps atomic elements of the centroid to atomic elements of the candidate on a domain-specific or generic relevance function. Over all relevances are aggregated using aggregate relevance functions. However, the requirements stated in Section 1 turn the development of generic solutions to this problem into a challenge.

\section{THE GRAPPA MATCHMAKING FRAMEWORK}

Figure 1 illustrates the structure of the GRAPPA matchmaking framework. It consists of three major parts. Its core is the matchmaking engine which computes the relevance between a multidimensional centroid and each element of a set of multidimensional candidates recurring into the sub-dimensions. It is complemented by the matchmaking library which contains generic and domain specific relevance functions for atomic and complex elements (see [8]). The matchmaking toolkit containing tools to specify centroid and candidate profile structures as well as mappings between these as XML DTDs completes the GRAPPA matchmaking framework. For details concerning the GRAPPA matchmaking framework see [8].

\section{APPLICATION: HRNetAgent}

The Human Resource Network (HRNetAgent) is an application of GRAPPA for matching multidimensional corporate job profiles with multidimensional profiles of job applicants (i.e., unemployed persons), stored in various data bases.

The backend of HRNetAgent consists of a collection of data sources wrapped by information agents, and by a search controller that coordinates a number of search agents.

The current version of HRNetAgent is a prototype system that has been developed for the German Labor Exchange Office, and demonstrates the feasibility of a partially automated approach to employment relaying. Based on its success, a full-fledged system is planned for the near future.

\section{CONCLUSIONS}

In this work, we have described a generic approach to matchmaking in agent- or human-mediated electronic marketplaces. The focus of this work is on achieving the flexibility and openness required to build a matchmaking framework that can be easily applied to different vertical marketplaces and that can be integrated into a broad range of industrial marketplace platforms and e-business systems.

The system currently provides only very basic feedback mechanisms that can be used to adapt the matchmaking configuration. We believe that learning capability will be required to achieve robust and good matchmaking behavior, and we are planning to incorporate feedback rules into the system in the future.

Finally, more elaborate methods are required to test the performance of complex matchmaking solutions.

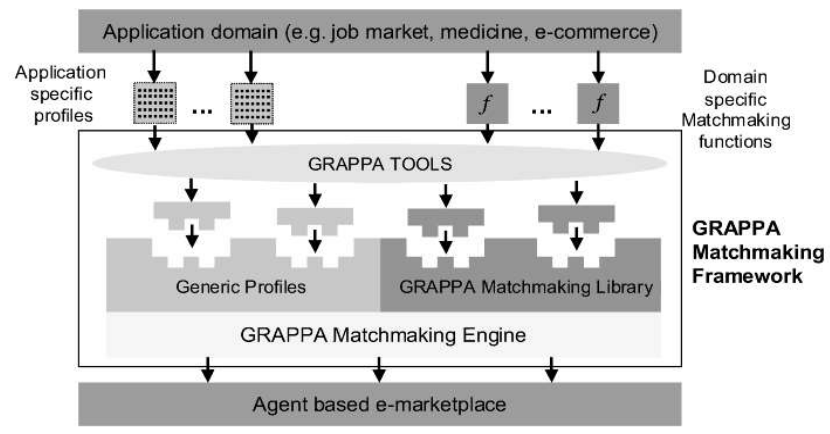

Figure 1. GRAPPA Matchmaking Framework.

\section{REFERENCES}

[1] Decker, K., Sycara, K., and Williamson, M. Matchmaking and Brokering. In International Conference on Multi-Agent Systems (ICMAS96), (1996, December).

[2] Kuokka, D. and Harada L. Integration information via matchmaking. Journal of Intelligent Information Systems 6(2/3), (1996), 261-279.

[3] Müller, J. P. and Pischel, M. Doing business in the information marketplace: a case study. In Proceedings of the $3^{\text {rd }}$ Intl. Conference on Autonomous Agents (Agents-1999), (1999), ACM Press.

[4] Nodine, M., Bohrer, W. and Ngu, A.H.H. Semantic brokering over dynamic heterogenous data sources in InfoSleuth. In Proceedings of the Fifteenth International Conference on Data Engeneering, (1999, August), 358-365.

[5] Sandholm, T. eMediator: a next generation electronic commerce server. In Proceedings of the $4^{\text {th }}$ Intl. Conference on Autonomous Agents (Agents-2000), (2000), 341-348, ACM Press.

[6] Subrahmanian, V.S., Bonatti, P., Dix, P., Eiter, T., Kraus, S., Ozcan, F. and Ross, R. Heterogenous Agent Systems. MIT Press. (ISBN: 0-262-19436-8), 2000.

[7] Sycara, K., Lu, J., Klusch, M., and Widoff, S. Dynamic service matchmaking among agents in open information environments. ACM SIGMOD Record 28 (1), Special Issue on Semantic Interoperability in Global Information Systems, (1999), 47-53.

[8] Veit, D. Matchmaking algorithms for autonomous agent systems. Master's Thesis, Institute of Computer Science, University of Giessen, Germany, (1999).

[9] Weinstein, P. and Birmingham, W. Service classification in a proto-organic society of agents. In Proceedings of the IJCAI97 Workshop on Artificial Intelligence in Digital Libraries, (1997).

[10] Wiederhold, G. Intelligent Integration of Information. In Proceedings of ACM SIGMOD Conference on Management of Data, Washington DC, (1993), 434-437. 